Research Paper

\title{
BDNF-mediates Down-regulation of MicroRNA-195 Inhibits Ischemic Cardiac Apoptosis in Rats
}

\author{
Pengzhou Hang ${ }^{1}$, Chuan Sun ${ }^{1}$, Jing Guo ${ }^{1}$, Jing Zhao², Zhimin Du ${ }^{1}$ \\ 1. Institute of Clinical Pharmacology of the Second Affiliated Hospital (Key Laboratory of Drug Research, Heilongjiang Higher Education Institutions), Harbin \\ Medical University, Harbin 150086, Heilongiiang Province, P. R. China; \\ 2. Department of Cardiology, the First Affiliated Hospital (Key Laboratory of Cardiac Diseases and Heart Failure), Harbin Medical University, Harbin 150001, \\ Heilongjiang Province, P. R. China. \\ $\triangle$ Corresponding author: Zhimin Du, PhD, Institute of Clinical Pharmacology of the Second Affiliated Hospital, Harbin Medical University, Xuefu Road 246, \\ Nangang District, Harbin 150086, Heilongjiang Province, P. R. China. Fax: +86 451 86665559, Tel: +86 451 86605353, Email: dzm1956@126.com.
}

() Ivyspring International Publisher. Reproduction is permitted for personal, noncommercial use, provided that the article is in whole, unmodified, and properly cited. See http://ivyspring.com/terms for terms and conditions.

Received: 2016.01.24; Accepted: 2016.06.07; Published: 2016.07.09

\begin{abstract}
Background: Our previous studies suggested that brain-derived neurotrophic factor (BDNF)/tropomyosin-related kinase B (TrkB) axis inhibited cardiomyocyte apoptosis in myocardial infarction (MI). However, the relationship between BDNF and microRNA (miRNA) in cardiomyocytes are unclear. The present study was performed to investigate the role of miR-195 and the interplay between BDNF and miR-195 in ischemic cardiomyocyte apoptosis.

Methods: Male Wistar rats were subjected to coronary artery ligation, and primary neonatal rat ventricular myocytes were treated with hypoxia or hydrogen peroxide $\left(\mathrm{H}_{2} \mathrm{O}_{2}\right)$. BDNF level in rat ventricles was measured by enzyme linked immunosorbent assay (ELISA). miR-195 mimic, inhibitor or negative control was transfected into the cardiomyocytes. Cell viability and apoptosis were detected by MTT assay and TdT-mediated dUTP nick end labeling (TUNEL) staining, respectively. Cardiac function and apoptosis were detected in MI rats intravenously injected with antagomiR-195. Luciferase assay, Western blot and Real-time RT-PCR were employed to clarify the interplay between miR-195 and BDNF.

Results: miR-195 level was dynamically regulated in response to $\mathrm{MI}$ and significantly increased in ischemic regions $24 \mathrm{~h}$ post-Ml as well as in hypoxic or $\mathrm{H}_{2} \mathrm{O}_{2}$-treated cardiomyocytes. Meanwhile, $\mathrm{BDNF}$ protein level was rapidly increased in $\mathrm{Ml}$ rats and $\mathrm{H}_{2} \mathrm{O}_{2}$-treated cardiomyocytes. Apoptosis in both hypoxic and $\mathrm{H}_{2} \mathrm{O}_{2}$-treated cardiomyocytes were markedly reduced and cell viability was increased by miR-195 inhibitor. Moreover, inhibition of miR-195 significantly improved cardiac function of MI rats. Bcl-2 but not BDNF was validated as the direct target of miR-195. Furthermore, BDNF abolished the pro-apoptotic role of miR-195, which was reversed by its scavenger TrkB-Fc.

Conclusion: Up-regulation of miR-195 in ischemic cardiomyocytes promotes ischemic apoptosis by targeting Bcl-2. BDNF mitigated the pro-apoptotic effect of miR-195 in rat cardiomyocytes. These findings may provide better understanding of the pro-apoptotic role of miR-195 in MI and suggest that BDNF/miR-195/Bcl-2 axis may be beneficial for limiting myocardial ischemic injury.
\end{abstract}

Key words: Brain-derived neurotrophic factor; miR-195; Myocardial ischemia; Apoptosis; Bcl-2.

\section{Introduction}

Acute myocardial infarction (MI) can lead to sudden cardiac death, which is one of the leading causes of death and disability worldwide [1]. Numerous studies have documented that cardiomyocyte apoptosis occurs in border zone of infarct scars and in the remote zone of non-infarcted myocardium [2-4], which exacerbates the post-MI remodeling and aggravates cardiac dysfunction [5]. Studies have also suggested that both acute substantial cardiomyocyte loss and chronic low levels 
of apoptosis contributed to the development of heart failure [6, 7]. Thus, successfully reversal of cardiomyocyte apoptosis during early stage of $\mathrm{MI}$ is crucial for repairing the injured heart and ameliorating cardiac function [8].

MicroRNAs (miRNAs or miRs) refer to a group of small noncoding RNA molecules, typically 18 25 nucleotides in length, that function in RNA silencing and post-transcriptional regulation of gene expression [9]. miR-195 is one of the members of miR-15 family including miR-15a, miR-15b, miR-16, miR-195 and miR-497 [10]. Recent studies elucidate that miR-195 plays a critical role in many cardiovascular diseases such as cardiac hypertrophy/heart failure, interstitial fibrosis and diabetic cardiomyopathy [11-13]. On the other hand, studies also suggested that miR-195 promoted apoptosis in a variety of cell types, e.g. hepatocellular carcinoma cells, neural progenitor cells, chondrocytes and cardiomyocytes [14-17]. At the same time, several apoptotic genes were screened as direct targets of miR-195, including Bcl-2, Sirtuin (Sirt1) and hypoxia-inducible factor (HIF)-1a according to bioinformatical prediction and experimental validation [16-18]. However, the role of miR-195 in cardiac ischemia-induced apoptosis and underlying mechanism is not fully understood.

Brain-derived neurotrophic factor (BDNF) is a member of neurotrophin factors, which is known to play key regulatory roles in neuronal activity and cognitive functions [19, 20]. Recently, BDNF is recognized to protect the heart by regulating the central nervous system [21] and promote neovascularization of ischemic tissue by recruiting the endothelial cells [22]. Our recent findings suggested that BDNF/tropomyosin-related kinase B (TrkB) axis possessed anti-ischemic apoptosis effect by increasing Bcl-2 expression and inhibiting caspase-3 activity [23]. Besides, BDNF was reported as a potential target of miR-195 based on previous studies [24]. Moreover, studies also revealed the link between BDNF and miRNAs in retinal ganglion cells and podocytes [25, 26]. Therefore, our present study focused on the role of miR-195, and the association between miR-195 and BDNF in ischemic cardiomyocyte apoptosis.

\section{Materials and methods}

\section{Ethics statement}

This study was approved by the Experimental Animal Ethic Committee of Harbin Medical University. All experimental procedures conformed to the Guide for the Care and Use of Laboratory Animals published by the US National Institutes of Health (Publication, 8th Edition, 2011).

\section{Rat MI model}

Male Wistar rats (weight $220 \pm 20 \mathrm{~g}$, provided by Animal Center of the Second Affiliated Hospital of Harbin Medical University, Harbin, China) were raised under standard animal room conditions (temperature $23 \pm 1{ }^{\circ} \mathrm{C}$; humidity $55 \pm 5 \%$ ) with free access of food and water. Rats were randomly divided into four groups: sham and MI for $1 \mathrm{~h}, 6 \mathrm{~h}$ or $24 \mathrm{~h}$. MI model was established by ligating the left anterior descending coronary artery as we previously described [23]. Sham-operated animals subjected to the same procedure but the coronary ligature was left untied. Electrocardiograms before, after ligation and at the end of ligation were recorded to confirm the ischemic condition. The surgery procedures were performed under sodium pentobarbital anesthesia. Remote, border and infarcted zones were quickly dissected for subsequent Real-time PCR analysis. To investigate the effect of miR-195 on ischemic heart function, antagomiR-195 (5 mg/kg, RiboBio Co., China) or negative control (NC) were injected via tail vein in the rats after the left anterior descending coronary artery was ligated. AntagomiR-195 is a single-stranded RNA analog complementary to the sequence of mature miR-195 (5'-UAGCAGCACAGA AAUAUUGGC-3') that is chemically modified and cholesterol-conjugated from a hydroxyprolinol-linked cholesterol solid support and 2'-OMe phosphoramidites. $24 \mathrm{~h}$ later, they were injected again at the same dose. 7 days post-MI, cardiac function was detected by echocardiography. To explore the role of BDNF on miR-195 in ischemic rat heart, recombinant human BDNF (10 $\mu \mathrm{g}$ in $25 \mu \mathrm{l}$ PBS, R\&D Systems, USA) and TrkB-Fc (5 $\mu \mathrm{g}$ in $25 \mu \mathrm{l} \mathrm{PBS,} \mathrm{688-TK-100,}$ TrkB-Fc chimera, R\&D Systems, USA) were intramuscular injected into the left ventricles according to previous studies [27]. $24 \mathrm{~h}$ post-MI border zones were collected.

\section{Echocardiography measurement and histological analysis}

Left ventricular function was measured by Acuson Sequoia 512 Ultrasound System (Siemens Medical Solutions USA, Inc., Mountain View, CA, USA). Left ventricular systolic diameter (LVDs), left ventricular diastolic diameter (LVDd) was measured, and left ventricular ejection fraction (LVEF) and fractional shortening (FS) were calculated. For histological analysis, border zones were embedded with paraffin, sectioned into $5 \mu \mathrm{m}$ slices. Then, they were stained with standard hematoxylin and eosin (HE).

\section{Cell culture and transfection}

Neonatal rat ventricular myocytes (NRVMs) 
were isolate and cultured according to previous study $[23,28]$. The isolated cells were cultured with DMEM (Corning, USA) and 10\% fetal bovine serum (ExCell Bio, China). The cardiomyocytes were cultured at $37^{\circ} \mathrm{C}$ with $5 \% \mathrm{CO}_{2}$ and $95 \%$ air. Cells were starved in serum-free medium for $12 \mathrm{~h}$, and then transiently transfected with miR-195 (50 nM), miR-195 inhibitor (100 nM) or negative control (NC), using X-treme GENE siRNA transfection reagent (Roche, USA) according to the manufacturer's instructions. miR-195 mimic, inhibitor and NC were synthesized by RiboBio Co., Ltd (China). The sequence of miR-195 mimic is 5'-UAGCAGCACAGAAAUAUUGGC-3', and mimic NC is $5^{\prime}$-UUUGUACUACACAAAAGUACUG-3'. The sequence of miR-195 inhibitor is 5'-GCCAAUAUUUC UGUGCUGCUA-3' and inhibitor NC is 5'-CAGUACUUUUGUGUAGUACAAA-3', which contains 2'-O-methyl modifications. 6h later, the culture medium were removed and DMEM containing serum was added to the cells. Cy3-labeled transfection control (Ribo Bio Co., Ltd, China) were used to validate the transfection efficiency. For hypoxic treatment, cells were placed in a hypoxic incubator (Thermo Scientific Series WJ 8000, USA) and were kept at $37^{\circ} \mathrm{C}$ for $12 \mathrm{~h}$ with a constant stream of water-saturated $94 \% \mathrm{~N}_{2}, 5 \% \mathrm{CO}_{2}$, and $1 \% \mathrm{O}_{2}$. Then, the cells were subsequently treated with different concentrations of hydrogen peroxide $\left(\mathrm{H}_{2} \mathrm{O}_{2}, 50\right.$ and $100 \mu \mathrm{M})$ for $4 \mathrm{~h}$ or $24 \mathrm{~h} . \mathrm{H}_{2} \mathrm{O}_{2}(100 \mu \mathrm{M}, 4 \mathrm{~h})$ was selected. Moreover, recombinant human BDNF (200 $\mathrm{ng} / \mathrm{ml}, \mathrm{R} \& D$ Systems, USA) and/or $4 \mu \mathrm{g} / \mathrm{ml}$ BDNF scavenger TrkB-Fc was treated to the cells as previously described [23]. Forty-eight hours after transfection, cardiomyocytes were collected for total RNA isolation or protein extraction.

\section{RNA extraction and Real-time PCR}

Total RNA were extracted from left ventricles of rats or NRVMs using Trizol reagent (Invitrogen, USA) according to manufacturer's protocols. RNA quantity was assessed using the NanoDropTM 8000 spectrophotometer (Thermo Scientific, France). cDNA was synthesized using reverse transcriptase kit (Roche, USA). Sequences of gene-specific PCR primers (Shanghai Generay Biotech Co. Ltd, China) used were as follow: miR-195 RT: 5'-GTCGTATCCAGTGCGTGTCGTGGAGTCGGCA ATTGCACTGGATACGACGCCAAT-3'; 5'-GGGGTAGCAGCAGCACAGAAAT-3';

5'-TCCAGTGCGTGTCGTGGA-3'; U6

5'-CGCTTCACGAATTTGCGTGTCAT-3';

5'-GCTTCGGCACATATACTAAAAT-3';

5'-CGCTTCACGAATTTGCGTGTCAT-3'. Primes of miR-15a, -15b, -16, -497 were listed in Supplementary Material: Table S1. Quantitative real-time PCR was performed in $20 \mu \mathrm{l}$ volumes with SYBR Green PCR Master Mix (Roche, USA) at $95^{\circ} \mathrm{C}$ for $10 \mathrm{~min}$ and 40 cycles at $95^{\circ} \mathrm{C}$ for $15 \mathrm{~s}, 60^{\circ} \mathrm{C}$ for $30 \mathrm{~s}$ and $72^{\circ} \mathrm{C}$ for $30 \mathrm{~s}$, using LightCycler 480 (Roche, USA). miR-195 levels were quantified with the $2-\Delta \Delta \mathrm{Ct}$ relative quantification method that was normalized to U6.

\section{BDNF level measurement}

BDNF level in ischemic zone of rat left ventricles was examined by enzyme linked immunosorbent assay (ELISA) according to the manufacturer's instructions (Promega, USA).

\section{TdT-mediated dUTP nick end labeling (TUNEL) staining}

TUNEL staining was employed to detect the apoptosis in left ventricles (border zones) and NRVMs as described in our previous study [23] using a TUNEL fluorescence FITC kit (Roche, USA) according to the manufacturer's instruction. After TUNEL staining, the cardiomyocytes were immerged into DAPI (1:30, Beyotime Biotechnology, China) solution to stain nuclei. Fluorescence staining was viewed by microscope (Olympus, BX-60, Japan) or a Laser Scanning Confocal Microscope (FV1000, Olympus, Japan). The apoptotic rate was calculated as TUNEL-positive cells per field.

\section{Cell viability assay}

MTT (3-[4, 5-dimethylthiazol-2-yl]-2, 5 diphenyl tetrazolium bromide) assay was used to examine the viability of cardiomyocytes. The cells were treated as designated and were subsequently incubated with 20 $\mu \mathrm{l}$ MTT $(0.5 \mathrm{mg} / \mathrm{ml})$ for $4 \mathrm{~h}$. Then, $200 \mu \mathrm{l}$ Dimethyl sulphoxide (DMSO) was added to each well to dissolve the formazan after removing the culture medium. The absorbance values were examined at $490 \mathrm{~nm}$ wavelength using an Infinite M200 microplate reader (Tecan, Salzburg, Austria) after rocking the cells for $10 \mathrm{~min}$.

\section{Luciferase activity assay}

The luciferase reporter assay was performed as previously described [29]. Briefly, luciferase reporters containing full-length wild-type 3'UTR of BDNF were constructed using pmiR-RB-REPORT ${ }^{\mathrm{TM}}$ vector. Then, 293T cells were seeded in a 24-well plate and co-transfected with $0.5 \mathrm{mg}$ plasmid and miR-195 mimic or NC by riboFectTM (Guangzhou Ribo Bio Co., Ltd). Renilla luciferase was used as an internal control. Forty-eight hours later, the cells were collected, and firefly and Renilla luciferase activities were measured in Dual-Glo $\AA$ Luciferase Assay System (Promega, Madison, WI, USA). 


\section{Western blot}

Total protein was extracted from the cardiomyocytes as described in previous studies [23, 29]. Briefly, proteins were separated by electrophoresis on SDS-polyacrylamide gels and transferred moist to nitrocellulose filter membranes. Membranes were incubated with anti-BDNF (A1307, 1:500, Abclonal, China), anti-TrkB (A2099, 1:500, Abclonal, China) or anti-Bcl-2 (ab7973, 1:500, Abcam, USA) antibody overnight at $4^{\circ} \mathrm{C}$. After washing, the membrane was incubated with IRDye secondary antibodies (LI-COR) for $1 \mathrm{~h}$. The images were captured by the Odyssey CLx Infrared Imaging System (LI-COR Biosciences, Lincoln, NE, USA). Western blot bands were quantified by measuring intensity in each group using Odyssey CLx version 2.1 with anti-GAPDH antibody (TA-09, 1:1000, ZSGB-Bio, China) as an internal control.

\section{Annexin V-FITC/propidium iodide (PI) staining assay}

An Annexin V-FITC Apoptosis Detection kit was used to examine early apoptosis (Annexin V-FITC+/PI-, Q4), late apoptosis (Annexin
A

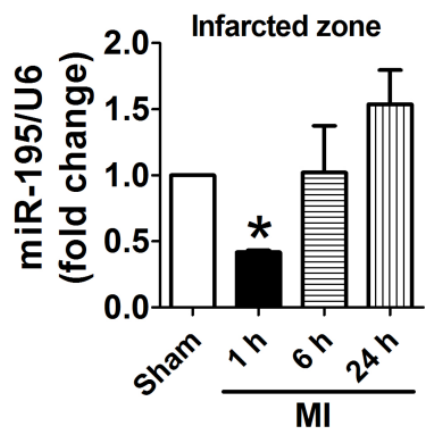

C



B

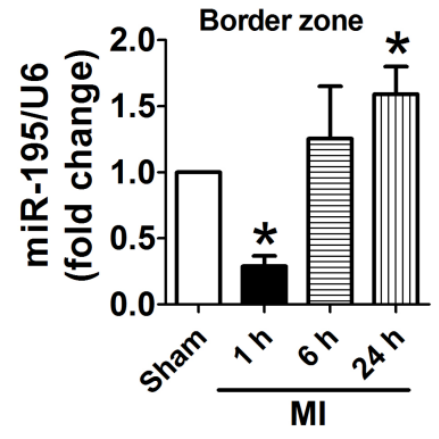

D

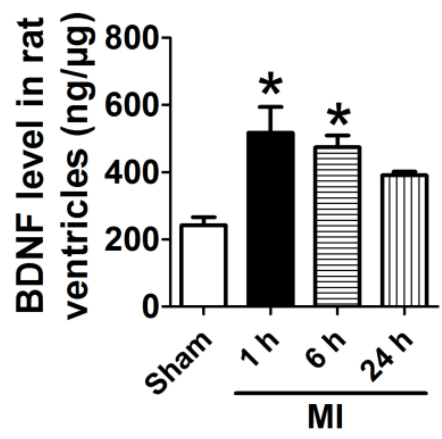

Figure 1. miR-195 and BDNF levels were dynamically regulated in different regions of rat myocardium in response to ischemic injury. (A, B) Real-time PCR analysis indicates that miR-195 is increased in the infarcted and border zones $24 \mathrm{~h}$ after myocardial infarction (MI). (C) miR-195 is decreased in remote zone in rat myocardium $24 \mathrm{~h}$ after MI. (D) BDNF level in ischemic zone of rat ventricles. ${ }^{*} \mathrm{p}<0.05,{ }^{*} \mathrm{p}<0.01$ vs. sham, $\mathrm{n}=$ 3.
V-FITC+/PI+, Q2), and necrosis (Annexin V-FITC-/PI+, Q1) according to the manufacturer's instructions (Vazyme, Nanjing, China). The cells were digested with $0.25 \%$ trypsin and collected by centrifugation after treatment. Then, the cells were washed twice with PBS and stained with Annexin V-FITC for $15 \mathrm{~min}$ and PI for $5 \mathrm{~min}$. The apoptotic cells were detected by flow cytometry (BD Bioscience, USA).

\section{Statistical analysis}

All data are expressed as mean \pm SEM and analyzed by GraphPad Prism 5.0 software. Two-group comparisons were performed by Student's $\mathrm{t}$ test. Multiple-group comparisons were carried out using one-way ANOVA followed by Dunnet's t-test. A two-tailed $P<0.05$ was considered to be significant.

\section{Results}

\section{miR-195 and BDNF were dynamically regulated in response to myocardial ischemic injury}

Because infarct healing is a dynamic process involving specific regional and temporal changes in cardiomyocytes, we first examined the expression of miR-195 in different zones after MI for $1 \mathrm{~h}, 6 \mathrm{~h}$ and $24 \mathrm{~h}$, respectively. Real-time PCR analysis revealed that miR-195 level was rapidly decreased in infarcted and border zones (Fig. 1A, B) whereas dramatically increased in remote zone $1 \mathrm{~h}$ after ischemic injury (Fig. 1C). Interestingly, miR-195 level was found to be elevated in infarcted and border zone while reduced in remote zone during the ischemic process. And miR-195 level was markedly higher in both infarcted and border zones $24 \mathrm{~h}$ after ischemic injury than sham rats (Fig. 1A, B). In contrast, it was significantly lower in remote zone of MI rats compared with sham rats (Fig. 1C). The expression of other miR-15 family members including miR-15a, miR-15b, miR-16, and miR-497 were also examined in infarcted, border and remote zones, respectively. As shown in Supplementary Material: Fig. S1, miR-15a, $-15 b$, -16 and -497 were all increased in $24 \mathrm{~h}$ post-MI compared with sham rats. Thus, the expression of these four miRNAs together with miR-195 had different amplitudes and characteristics in response to $\mathrm{MI}$ although belonging to the same family. Meanwhile, BDNF level in ischemic myocardium was found rapidly increased at $1 \mathrm{~h}$ and $6 \mathrm{~h}$ post-MI and recovered $24 \mathrm{~h}$ post-MI (Fig. 1D). Thus, both miR-195 and BDNF are 
dynamically regulated in response to MI.

\section{miR-195 mimic and inhibitor transfection validation}

Cy3-labeled transfection control was used to examine the transfection efficiency of miR-195 mimic and inhibitor. As displayed in Supplementary Material: Fig. S2, Cy3-labeled transfection control (red color) was widely distributed around the nuclear (stained in blue with DAPI), which suggested that the transfection efficiency satisfied with experimental requirements. Then, to overexpress or knockdown miR-195 expression, miR-195 mimic or inhibitor was transfected into the cardiomyocytes. We further validate the miR-195 level using Real-time PCR. We found that miR-195 level was significantly increased after transfecting with miR-195 mimic ( $\sim 557$ folds, $\mathrm{p}<0.01$, Fig. 2A). Moreover, miR-195 was markedly decreased in miR-195 inhibitor transfection group compared with non-transfection group ( $\sim 0.28$ fold, $\mathrm{p}<0.001$, Fig. 2B). These findings strongly supported that miR-195 mimic or inhibitor were successfully transfected into the cardiomyocytes to upregulate or downregulate miR-195, respectively.

\section{miR-195 inhibitor prevented hypoxia-induced cardiomyocyte apoptosis}

Cardiomyocytes were subjected to hypoxia to simulate ischemic status in vitro. Consistently, we found that the miR-195 level was significantly higher in hypoxic cells than normoxia cells (Fig. 3A). In addition, TUNEL assay was used to detect the role of miR-195 in hypoxia-induced cardiomyocyte apoptosis. It was observed that the TUNEL-positive cells were significantly increased in hypoxia group, which was diminished by miR-195 inhibitor but not by miR-NC (Fig. 3B, C). These data implied that miR-195 inhibitor prevented hypoxia-induced cardiomyocyte apoptosis.
A

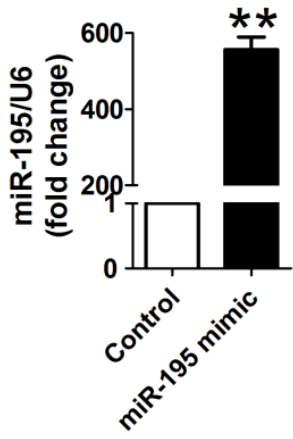

B

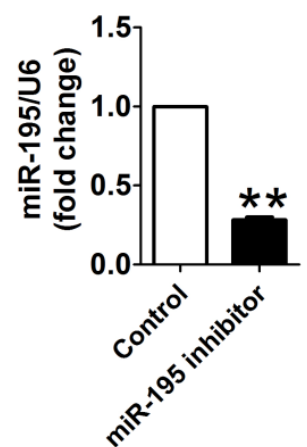

Figure 2. miR-195 mimic and inhibitor transfection validation. (A) Real-time PCR analysis indicated that miR-195 level was dramatically increased after transfecting with miR-195 mimic compared with control group. (B) miR-195 level was markedly decreased after transfecting with miR-195 inhibitor compared with control group. ${ }^{* *} \mathrm{p}<0.01$ vs. control, $* * * p<0.001$ vs. control, $\mathrm{n}=3$.
A



B

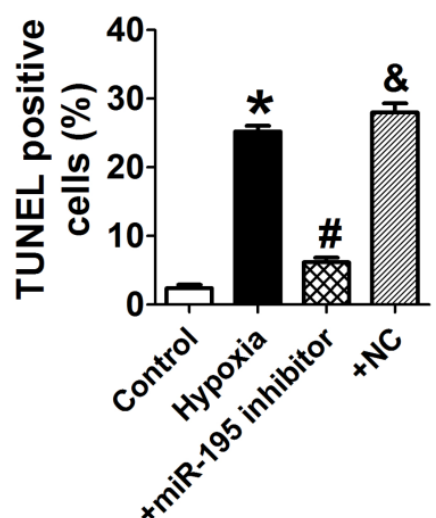

C<smiles>[Mg][Mg][Mg]</smiles>
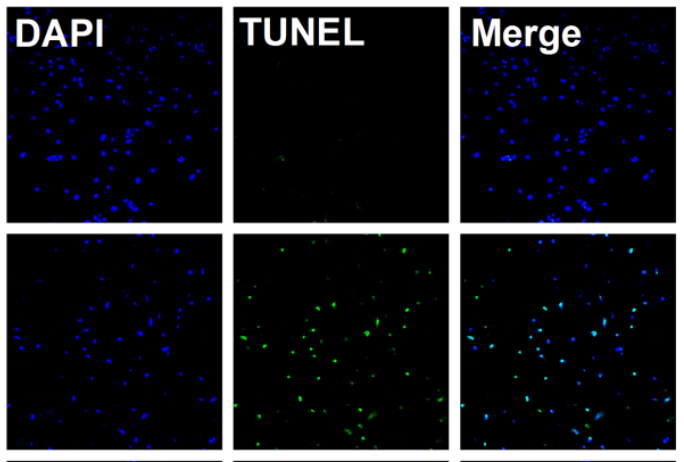

+miR-195 inhibitor
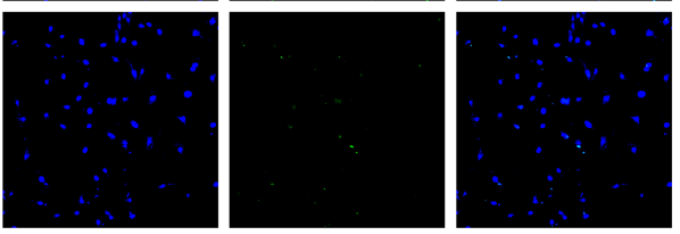

$+\mathrm{NC}$
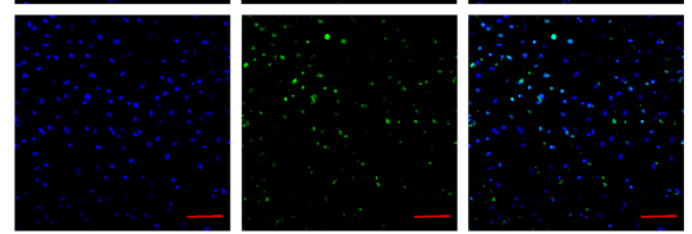

Figure 3. miR-195 inhibitor attenuated hypoxia-induced cardiomyocyte apoptosis. (A) Real-time PCR analysis indicates that miR-195 is increased in hypoxia-treated cardiomyocytes. (B) Statistical results of TUNEL-positive cells per field. (C) Representative images of TUNEL staining of cardiomyocyte showing the apoptotic cells (apoptotic cells stained in green and nucleus stained in blue with DAPI). ${ }^{*} \mathrm{p}<0.05$ vs. control, \#p<0.05 vs. hypoxia, $\mathrm{n}=5$, scale bar: $100 \mu \mathrm{m}$. 
A

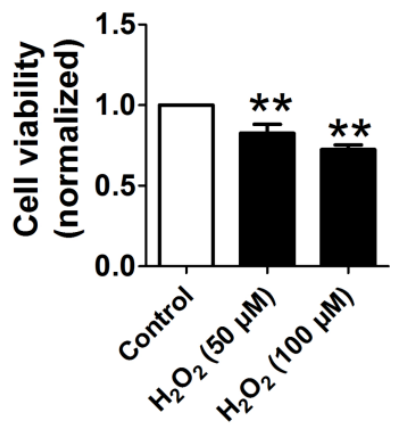

B

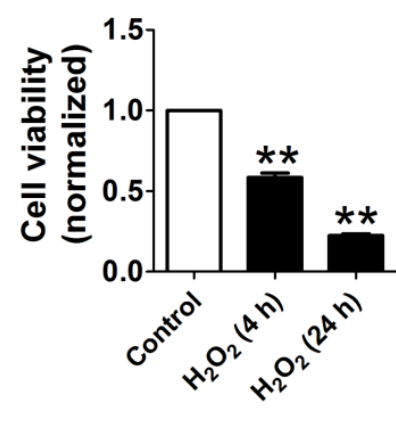

C

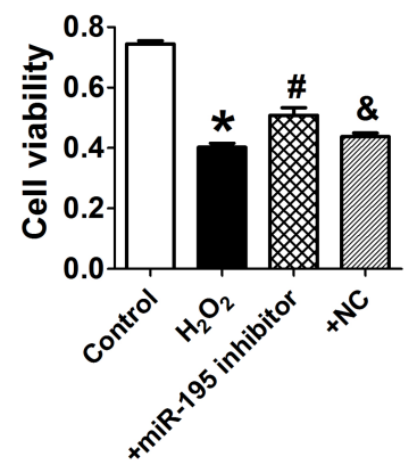

Figure 4. miR-195 inhibitor protected against cardiomyocyte injury induced by hydrogen peroxide $\left(\mathrm{H}_{2} \mathrm{O}_{2}\right)$. $(\mathrm{A}$, B) Cultured neonatal rat cardiomyocytes were exposed to different concentration of of $\mathrm{H}_{2} \mathrm{O}_{2}(50,100 \mu \mathrm{M})$ for $4 \mathrm{~h}$, or different timepoints $(4 \mathrm{~h}, 24 \mathrm{~h})$ at $100 \mu \mathrm{M}$. (C) MTT assay suggested that miR-195 inhibitor restored cell viability after $\mathrm{H}_{2} \mathrm{O}_{2}$ treatment. ${ }^{*}<<0.05$, ${ }^{* *} \mathrm{p}<0.01$ vs Control, $\# \mathrm{p}<0.05$ vs $\mathrm{H}_{2} \mathrm{O}_{2}$, \& $p<0.05$ vs miR-195 inhibitor, $n=5$.

A

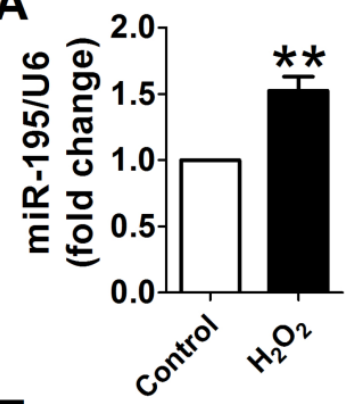

E

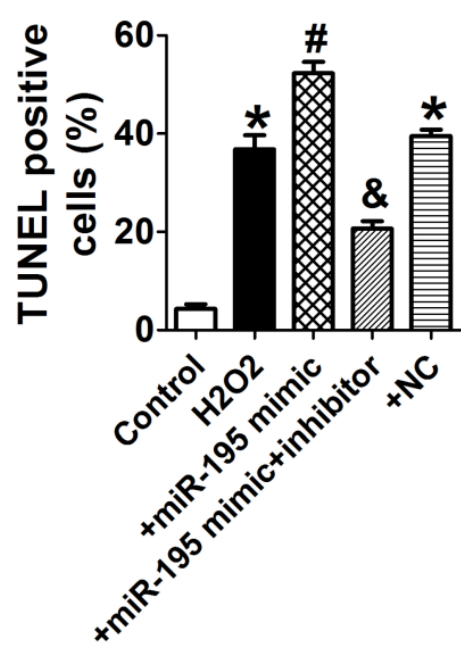

B

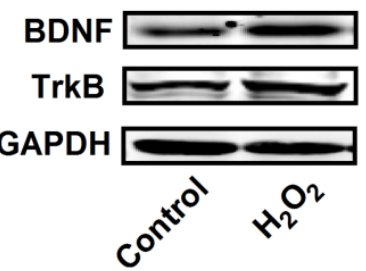

$\mathbf{F}$
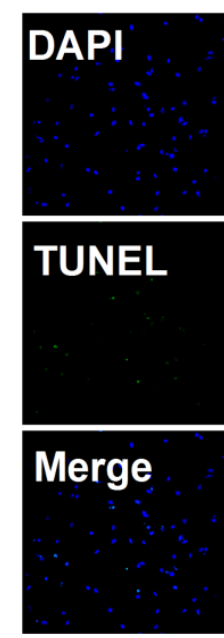

Control
2000

nowase

stose

C

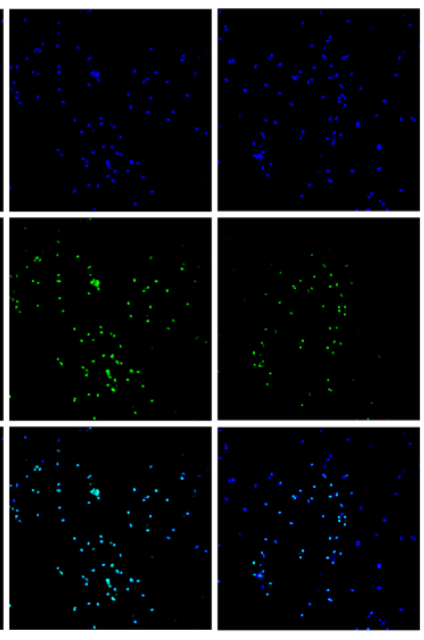

$\mathrm{H}_{2} \mathrm{O}_{2}+$ miR-195 mimic +miR-195 mimic+inhibitor

Figure 5. miR-195 inhibitor attenuated $\mathrm{H}_{2} \mathrm{O}_{2}$-induced cardiomyocyte apoptosis. (A) Real-time PCR analysis indicates that miR-195 is increased in $\mathrm{H}_{2} \mathrm{O}_{2}$-treated cardiomyocytes. (B) Western blot bands of BDNF and TrkB in control and $\mathrm{H}_{2} \mathrm{O}_{2}$-treated cardiomyocytes. (C, D) Statistical results of protein level of BDNF and TrkB, $* p<0.05, n=5$. (E) Statistical results of TUNEL-positive cells per field. (F) Representative images of TUNEL staining of cardiomyocyte showing the apoptotic cells (apoptotic cells stained in green and nucleus stained in blue with DAPI). ${ }^{*} \mathrm{p}<0.05$ vs. control, $\# \mathrm{p}<0.05 \mathrm{vs}$. $\mathrm{H}_{2} \mathrm{O}_{2}, \& \mathrm{p}<0.05 \mathrm{vs}$. + miR-195 mimic, $\mathrm{n}=5$, scale bar: $100 \mu \mathrm{M}$.

\section{miR-195 inhibitor prevented $\mathrm{H}_{2} \mathrm{O}_{2}$-induced cardiomyocyte apoptosis}

Consistent with previous study [28], cell viability was significantly decreased by $50 \mu \mathrm{M}$ and $100 \mu \mathrm{M}$ $\mathrm{H}_{2} \mathrm{O}_{2}$ for $4 \mathrm{~h}$, meanwhile markedly decreased after $\mathrm{H}_{2} \mathrm{O}_{2}$ treatment for $4 \mathrm{~h}$ and $24 \mathrm{~h}$ at $100 \mu \mathrm{M}$ (Fig. 4A, B). Accordingly, $\mathrm{H}_{2} \mathrm{O}_{2}$ reduced cardiomyocyte viability in a concentration and time-dependent manner and 100 $\mu \mathrm{M} \mathrm{H}_{2} \mathrm{O}_{2}$ treated for $4 \mathrm{~h}$ was chosen for subsequent experiments. We found that cell viability treated with $\mathrm{H}_{2} \mathrm{O}_{2}$ was restored by miR-195 inhibitor but not by miR-NC (Fig. 4C). In agreement with hypoxia, miR-195 level was found to be increased in $\mathrm{H}_{2} \mathrm{O}_{2}$-treated cardiomyocytes compared with control cells (Fig. 5A). Western blot analysis indicated that BDNF and TrkB were significantly increased in 
$\mathrm{H}_{2} \mathrm{O}_{2}$-treated cardiomyocytes (Fig. 5B-D). Furthermore, we found that the TUNEL-positive cells were significantly increased by miR-195 mimic in $\mathrm{H}_{2} \mathrm{O}_{2}$-treated group, which was reversed by miR-195 inhibitor but not by miR-NC (Fig. 5E, F). These results together suggested that miR-195 is pro-apoptotic in $\mathrm{H}_{2} \mathrm{O}_{2}$-treated cardiomyocytes. Meanwhile, knockdown of endogenous miR-195 prevented $\mathrm{H}_{2} \mathrm{O}_{2}$-induced apoptosis of cardiomyocytes.

\section{AntagomiR-195 improved cardiac function in MI rats}

EF and FS were markedly decreased in MI+NC rats compared with sham rats, which was significantly restored by antagomiR-195 (Fig. 6A, B). Meanwhile, MI-induced increment of LVDs and LVDd were reduced in antagomiR-195 rats (Fig. 6C, D). HE staining displayed that cardiac injury induced by $\mathrm{MI}$ was markedly attenuated by antagomiR-195 but not by NC (Fig. 7A). Moreover, the role of miR-195 in MI-induced cardiomyocyte apoptosis was also validated in rats. As shown in Fig. 7B, C, TUNEL positive cells were markedly decreased in antogomiR-195-treated MI rats compared with $\mathrm{MI}+\mathrm{NC}$ rats. Consistently, Bcl-2 protein expression was found upregulated by antagomiR-195 (Fig. 7D, E). So, these data together supported that inhibition of miR-195 improved cardiac function in MI.

\section{Interplay between miR-195 and BDNF}

We further examined the possible interaction between miR-195 and BDNF. As illustrated in Fig. 8A, the 3'UTR of $B d n f$ has one binding site of miR-195. We detected the protein expression of BDNF after transfected with miR-195 mimic or inhibitor. And no significant change was observed among control, miR-195 mimic and miR-195 inhibitor groups (Fig. 8B, C). Then, luciferase assay was further employed to validate the regulatory effect of miR-195 on BDNF. Consistent to western blot results, no significant change of luciferase reporter activity was found between miR-195 mimic and NC (Fig. 8D). These findings demonstrated that BDNF is not a direct target of miR-195. Besides, previous bioinformatical analysis and experimental studies have proved the anti-apoptotic factor Bcl-2 was a direct target of miR-195 [18]. In our present study, we found that protein expression of $\mathrm{Bcl}-2$ was significantly inhibited by miR-195 mimic (Fig. 8E, F), and validated the relatioship between miR-195 and Bcl-2. On the other hand, we tried to clarify the effect of BDNF on miR-195 by detecting miR-195 level after administering with BDNF or its blocker TrkB-Fc in both rats and NRVMs. We found that miR-195 level was obviously repressed by BDNF, which could be antagonized by TrkB-Fc both in vivo and in vitro (Fig. 9A, B). Next, we found that BDNF increased cell viability $\mathrm{H}_{2} \mathrm{O}_{2}$ treatment and was reversed by TrkB-FC (Fig. 9C). Finally, flow cytometry was utilized to validate the protective role of BDNF. We found that the apoptosis rate was increased by $\mathrm{H}_{2} \mathrm{O}_{2}$ and diminished by BDNF, which was reversed by TrkB-Fc (Fig. 9D, E). Taken together, these findings suggested that BDNF inhibited miR-195 expression and prevented cardiomyocyte apoptosis.

\section{Discussion}

The present study demonstrated that miR-195 was up-regulated in both ischemic myocardium and hypoxia $/ \mathrm{H}_{2} \mathrm{O}_{2}$-induced cardiomyocytes. Up-regulation of miR-195 in ischemic cardiomyocytes promotes ischemic apoptosis by targeting Bcl-2. BDNF mitigated the pro-apoptotic effect of miR-195 in cardiomyocytes. These findings suggest that $\mathrm{BDNF} / \mathrm{miR}-195 / \mathrm{bcl}-2$ axis may be beneficial for repairing the ischemic cardiomyocytes.
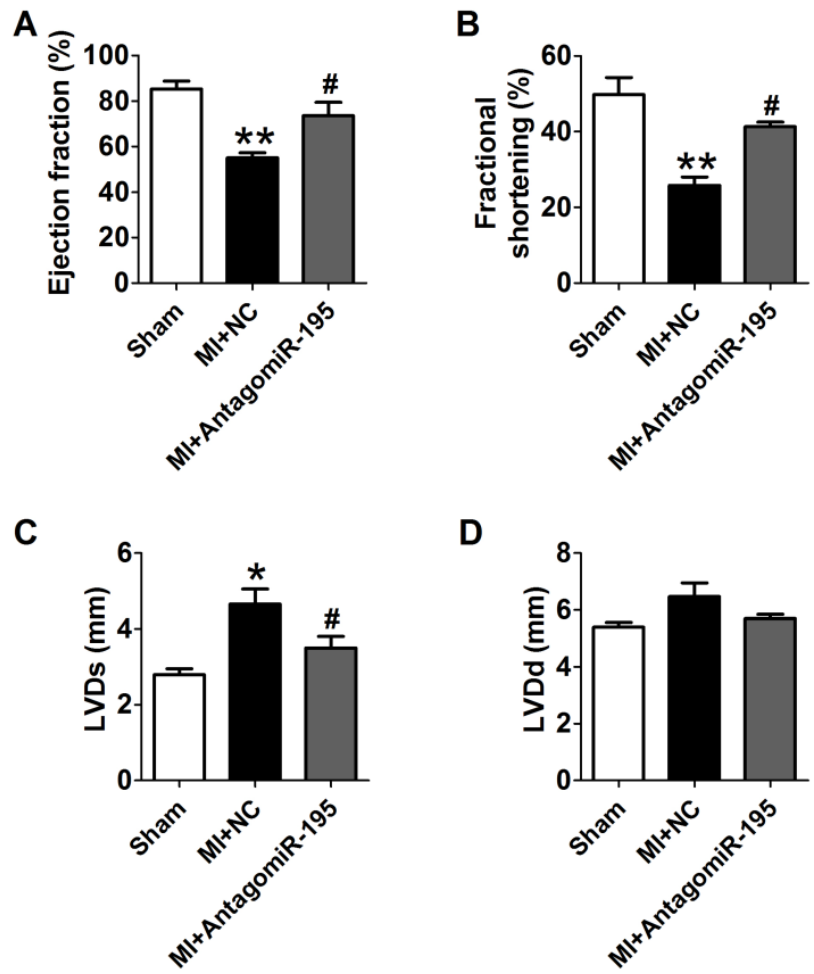

Figure 6. Improvement of cardiac function by antagomiR-195 in MI rats. (A) Ejection fractions. (B) Fractional shortening. (C) Left ventricular systolic diameter (LVDs). (D) Left ventricular diastolic diameter (LVDd). ${ }^{*} p<0.05$, $* * \mathrm{p}<0.01$ vs. sham, $\# \mathrm{p}<0.05$ vs. $\mathrm{MI}+\mathrm{NC}, \mathrm{n}=5$. 
A


B
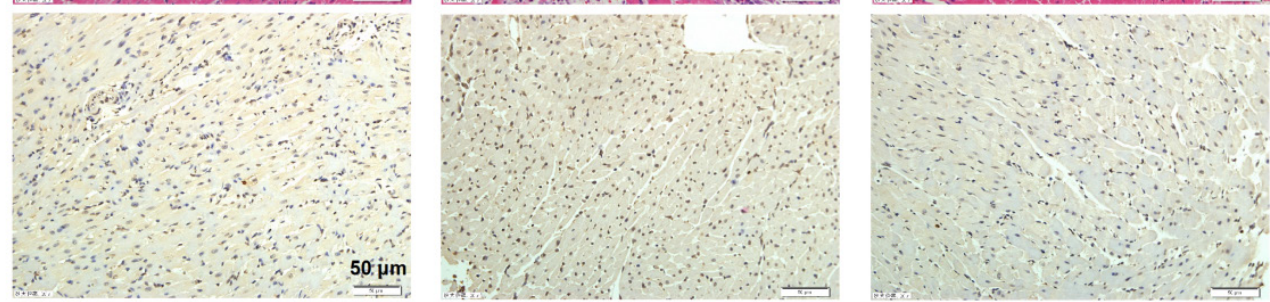

Sham

MI+NC

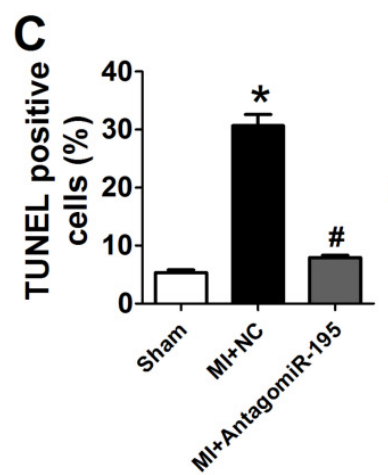

D

\section{MI+AntagomiR-195}

$\mathrm{Bcl}-2$

GAPDH
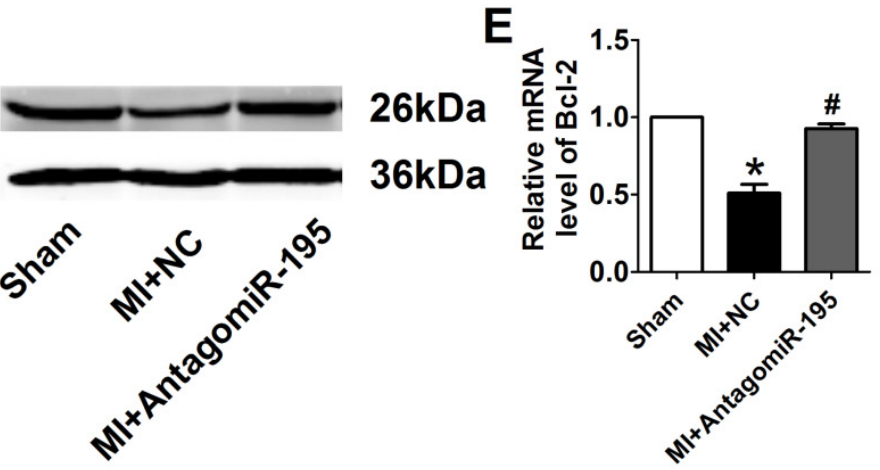

Figure 7. AntagomiR-195 inhibited cardiac injury and apoptosis by upregulating $\mathrm{Bcl}-2$ in $\mathrm{Ml}$ rats. (A) Representative $\mathrm{HE}$ staining pictures, scale bar: $50 \mu \mathrm{M}$. (B) Representative images of TUNEL staining in rat myocardium (apoptotic cells stained in brown), scale bar: $50 \mu M$. (C) Statistical results of TUNEL-positive cells per field. (D) Western blot bands of Bcl-2 in sham, MI+NC and Ml+antagomiR-195 rat hearts. (E) Statistical results of protein level of Bcl-2. *p<0.05 vs. sham, \#p<0.05 vs. $M I+N C, n=5$.

$$
\begin{aligned}
& \text { A } \\
& 3 \text { ' -CGGUUAUAAAGAC-ACGACGAU- } 5 \text { ' }
\end{aligned}
$$

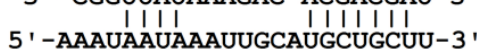

$$
\begin{aligned}
& \text { 292-298 of Bdnf 3' UTR } \\
& \text { hsa-miR-195 } \\
& \text { 3'-CGGUUAUAAAGAC-ACGACGAU-5 ' } \\
& 5 \text { '-AAAUAAUAAAUUGCAUGCUGCUU-3' } \\
& \text { 300-306 of Bdnf 3' UTR }
\end{aligned}
$$

D

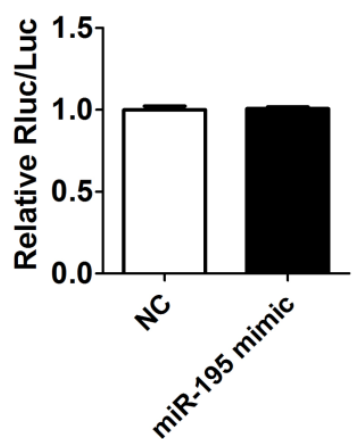

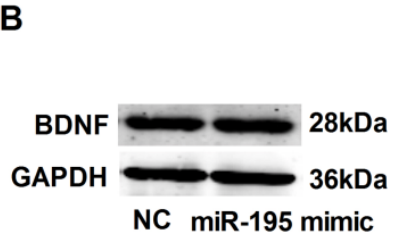

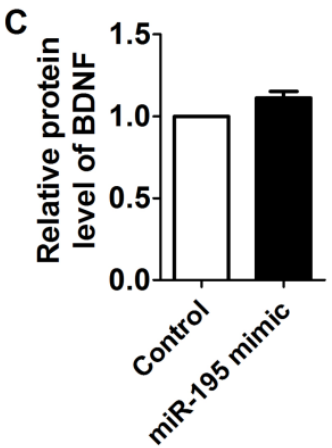

E

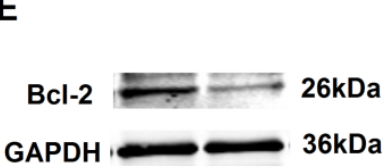

NC miR-195 mimic

F

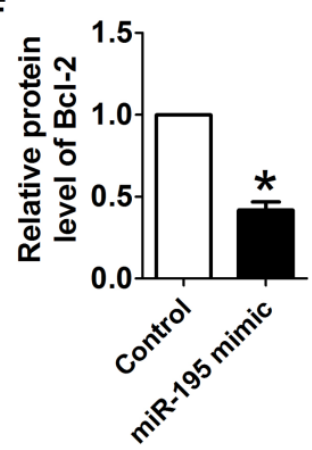

Figure 8. Target validation of miR-195. (A) Sequence alignment show between miR-195 and the binding sites in the 3'UTR of the Bdnf gene. (B) Representative western blot bands of BDNF. (C) Statistical results of protein level of BDNF in miR-195 mimic and NC group, $n=3$. (D) The interaction between miR-195 and its binding sites in the 3'UTR of Bdnf was examined by luciferase assay in HEK293 cells, $n=3$. (E) Representative western blot bands of Bcl-2. (F) Statistical results of protein level of $\mathrm{Bcl}-2$ in miR-195 mimic and NC group, ${ }^{\mathrm{p}}<0.05$, vs. control, $\mathrm{n}=3$. 
A
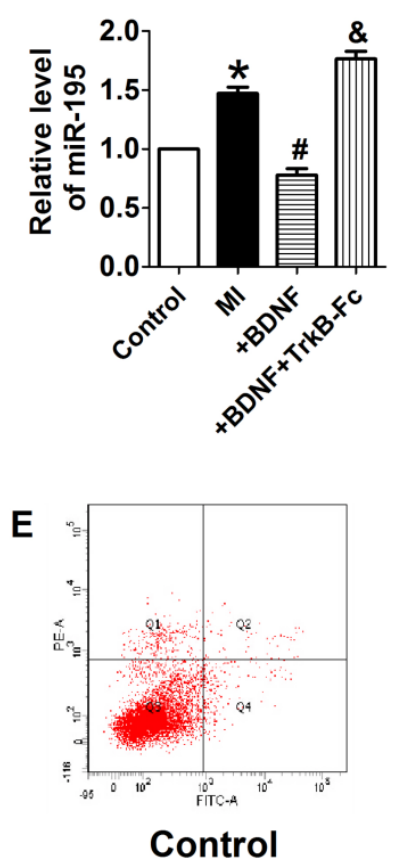

B
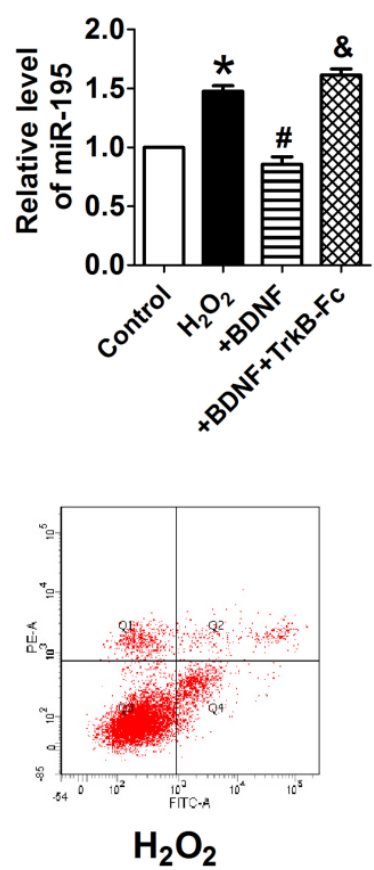

C

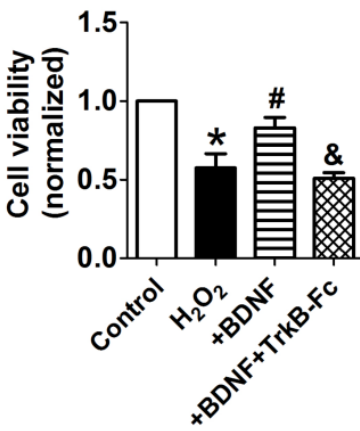

D
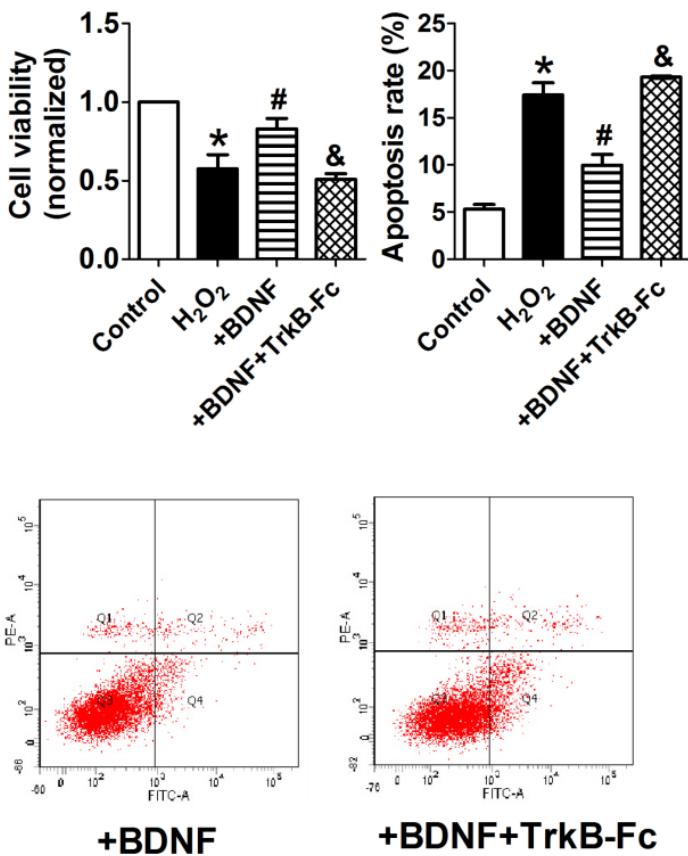

Figure 9. BDNF inhibited miR-195 expression and protected cardiomyocytes against $\mathrm{H}_{2} \mathrm{O}_{2}$-induced apoptosis. (A) Real-time PCR analysis indicates that miR-195 level is reduced by BDNF and restored by TrkB-Fc, ${ }^{*} p<0.05$, vs. control, $\# p<0.05$ vs $\mathrm{H}_{2} \mathrm{O}_{2}$, \& $p<0.05$ vs $+B D N F, n=5$. (B) $M T T$ assay showed that $B D N F$ improved cell viability in $\mathrm{H}_{2} \mathrm{O}_{2}$-treated cardiomyocytes and was reversed by TrkB-Fc, ${ }^{*} p<0.05$, vs. control, \#p<0.05 vs $\mathrm{H}_{2} \mathrm{O}_{2}, \& p<0.05$ vs $+\mathrm{BDNF}, \mathrm{n}=5$. (C) The quantitative presentation of apoptotic cells by Annexin V-FITC/propidium iodide $(\mathrm{PI})$ staining, ${ }^{*} \mathrm{p}<0.05$, vs. control, $\# p<0.05$ vs $\mathrm{H}_{2} \mathrm{O}_{2}, \& \mathrm{p}<0.05$ vs $+\mathrm{BDNF}, \mathrm{n}=3$. $(\mathrm{D})$ Representative Annexin V-FITC/PI staining pictures.

The regulation of miR-195 is spatial, temoral and dynamic, and its expression profile depends on species and pathological stress [30-32]. In an earlier study by Hullinger et al. [30], miR-195 was found to be up-regulated in the infarcted zone $24 \mathrm{~h}$ after ischemic-reperfusion (IR) injury in the porcine MI model but did not significantly increased in the border zone. Another study reported that miR-195 was downregulated in both border and infarct zone of mice in response to MI [31]. In contrast, we noted that miR-195 expression elevated in both infarcted and border zones and reduced in remote zone in response to MI. Moreover, it has also been reported that only miR-15b but no other miR-15 family members was upregulated in mouse heart subjected to IR for 2 or 7 days [32]. These different results may at least partly be explained by distinct species and animal models.

We focused on miR-195, as this miR has been previously reported as a miR with potential pro-apoptotic properties [14-17], but to the best of our knowledge, the present study firstly demonstrated the critical role of miR-195 in ischemic apoptosis and heart function. In previous studies, miR-195 mimic inhibited the protein level of $\mathrm{Bcl}-2$ and contributed to podocyte apoptosis by increasing caspase- 3 in high-glucose culture podocytes [33]. In cardiomyocytes, up-regulation of miR-195 expression promoted apoptosis and ROS production after palmitate-stimulation [17]. In line with these studies, our data demonstrated that knockdown of miR-195 alleviated cardiomyocyte apoptosis. Considering several previous studies have proved that Bcl-2 was an important direct target of miR-195 [17, 18]. Only western blot was employed to validate the effect of miR-195 mimic on Bcl-2 protein expression in the cardiomyocytes.

There is controversy in regarding of whether BDNF is the direct target of miR-195. Mellios et al. proposed that miR-195 potentially target 3'-untranslated region of BDNF using luciferase assays [24]. But they did not validate the protein expression of BDNF after transfection of miR-195 mimic. On the contrary, recent studies pointed out that BDNF is not the target of miR-195 according to luciferase assay results [34-36]. To clarify this question, both luciferase assay and western blot experiments were employed in the present study. And our data suggested that transfection with miR-195 mimic could not reduce luciferase report activity. Consistently, neither miR-195 mimic nor miR-195 inhibitor changed the protein expression of BDNF. Therefore, our findings supported that BDNF is not a direct target of miR-195. On the other hand, protein level of BDNF was also dynamically regulated (rapidly increased after $1 \mathrm{~h}$ ischemia and gradually decreased after $24 \mathrm{~h}$ ischemia) in response to ischemic 
injury. Interestingly, we noted an inverse relationship between BDNF and miR-195 expression. So we examined the effect of BDNF on miR-195 and found that miR-195 was negatively regulated by BDNF. Our previous study has proved that BDNF treatment up-regulated Bcl-2 expression [23], which is widely accepted as a direct target of miR-195 [18]. Incorporating with these studies, we speculated the protective role of $\mathrm{BDNF} / \mathrm{miR}-195 / \mathrm{Bcl}-2$ axis against ischemic apoptosis. Likewise, we noted that previous studies by Iekushi et al. demonstrated that bone marrow-derived mononuclear cells released insulin-like growth factor-1 (IGF-1), and prevented cardiomyocyte apoptosis by blocking expression of the precursor and the mature miR-34a [37]. This study indicates that the effect of cytokines/growth factor on miRNAs maybe a general phenomenon. Such possible interactions may provide further evidence for exploring upstream of miRNAs in cardiomyocytes.

In conclusion, this study suggested that BDNF-mediated down-regulation of miR-195 inhibited ischemic cardiomyocyte apoptosis, which may provide novel idea for treatment of ischemic heart diseases.

\section{Supplementary Material}

Supplementary tables and figures.

http://www.ijbs.com/v12p0979s1.pdf

\section{Acknowledgments}

This work was supported in part by Major Program of National Natural Science Foundation of China (81230081), National Natural Science Foundation of China (81300080, 81200077), Natural Science Foundation of Heilongjiang Province (QC2013C087), and Postgraduate Innovation Foundation of Harbin Medical University (YJSCX2015-9HYD).

\section{Competing Interests}

The authors have declared that no competing interest exists.

\section{References}

1. Mozaffarian D, Benjamin EJ, Go AS, Arnett DK, Blaha MJ, Cushman M et al. Heart Disease and Stroke Statistics-2016 Update: A Report From the American Heart Association. Circulation. 2016; 133: e38-e360.

2. Saraste A, Pulkki K, Kallajoki M, Henriksen K, Parvinen M, Voipio-Pulkki LM. Apoptosis in human acute myocardial infarction. Circulation. 1997; 95: $320-323$.

3. Olivetti G, Quaini F, Sala R, Lagrasta C, Corradi D, Bonacina E, Gambert SR, Cigola E, Anversa P. Acute myocardial infarction in humans is associated with activation of programmed myocyte cell death in the surviving portion of the heart J Mol Cell Cardiol 1996: 28: 2005-2016.

4. Piro FR, di Gioia CR, Gallo P, Giordano C, d'Amati G. Is apoptosis a diagnostic marker of acute myocardial infarction? Arch Pathol Lab Med. 2000; 124: $827-831$.

5. Palojoki E, Saraste A, Eriksson A, Pulkki K, Kallajoki M, Voipio-Pulkki LM, Tikkanen I. Cardiomyocyte apoptosis and ventricular remodeling after myocardial infarction in rats. Am J Physiol Heart Circ Physiol. 2001; 280: H2726-H2731.
6. Narula J, Haider N, Virmani R, DiSalvo TG, Kolodgie FD, Hajjar RJ, Schmidt U, Semigran MJ, Dec GW, Khaw BA. Apoptosis in myocytes in end-stage heart failure. N Engl J Med. 1996; 335: 1182-1189.

7. Wencker D, Chandra M, Nguyen K, Miao W, Garantziotis S, Factor SM, Shirani J, Armstrong RC, Kitsis RN. A mechanistic role for cardiac myocyte apoptosis in heart failure. J Clin Invest. 2003; 111: 1497-1504.

8. Eltzschig HK, Eckle T. Ischemia and reperfusion--from mechanism to translation. Nat Med. 2011; 17: 1391-1401.

9. Boon RA, Dimmeler S. MicroRNAs in myocardial infarction. Nat Rev Cardiol. 2015; 12: 135-142.

10. Finnerty JR, Wang WX, Hébert SS, Wilfred BR, Mao G, Nelson PT. The miR-15/107 group of microRNA genes: evolutionary biology, cellular functions, and roles in human diseases. J Mol Biol. 2010; 402: 491-509.

11. van Rooij E, Sutherland LB, Liu N, Williams AH, McAnally J, Gerard RD, Richardson JA, Olson EN. A signature pattern of stress-responsive microRNAs that can evoke cardiac hypertrophy and heart failure. Proc Natl Acad Sci U S A. 2006; 103: 18255-18260.

12. Tijsen AJ, van der Made I, van den Hoogenhof MM, Wijnen WJ, van Deel ED, de Groot NE, Alekseev S, Fluiter K, Schroen B, Goumans MJ, van der Velden J, Duncker DJ, Pinto YM, Creemers EE. The microRNA-15 family inhibits the TGFß-pathway in the heart. Cardiovasc Res. 2014; 104: 61-71.

13. Zheng D, Ma J, Yu Y, Li M, Ni R, Wang G, Chen R, Li J, Fan GC, Lacefield JC, Peng T. Silencing of miR-195 reduces diabetic cardiomyopathy in C57BL/6 mice. Diabetologia. 2015; 58: 1949-1958.

14. Yang X, Yin J, Yu J, Xiang Q, Liu Y, Tang S, Liao D, Zhu B, Zu X, Tang H, Lei X. miRNA-195 sensitizes human hepatocellular carcinoma cells to 5 -FU by targeting BCL-w. Oncol Rep. 2012; 27: 250-257.

15. Zhou Y, Jiang H, Gu J, Tang Y, Shen N, Jin Y. MicroRNA-195 targets ADP-ribosylation factor-like protein 2 to induce apoptosis in human embryonic stem cell-derived neural progenitor cells. Cell Death Dis. 2013; 4: e695.

16. Bai $\mathrm{R}$, Zhao AQ, Zhao ZQ, Liu WL, Jian DM. MicroRNA-195 induced apoptosis in hypoxic chondrocytes by targeting hypoxia-inducible factor 1 alpha. Eur Rev Med Pharmacol Sci. 2015; 19: 545-551.

17. Zhu H, Yang Y, Wang Y, Li J, Schiller PW, Peng T. MicroRNA-195 promotes palmitate-induced apoptosis in cardiomyocytes by down-regulating Sirt1. Cardiovasc Res. 2011; 92: 75-84.

18. Liu L, Chen L, Xu Y, Li R, Du X. microRNA-195 promotes apoptosis and suppresses tumorigenicity of human colorectal cancer cells. Biochem Biophys Res Commun. 2010; 400: 236-240.

19. Wong $\mathrm{YH}$, Lee CM, Xie W, Cui B, Poo MM. Activity-dependent BDNF release via endocytic pathways is regulated by synaptotagmin- 6 and complexin. Proc Natl Acad Sci U S A. 2015; 112: E4475-E4484

20. Ma JC, Duan MJ, Sun LL, Yan ML, Liu T, Wang Q, Liu CD, Wang X, Kang XH, Pei SC, Zong DK, Chen $\mathrm{X}$, Wang $\mathrm{N}$, Ai J. Cardiac over-expression of microRNA-1 induces impairment of cognition in mice. Neuroscience. 2015; 299: 66-78.

21. Okada S, Yokoyama M, Toko H, Tateno K, Moriya J, Shimizu I, Nojima A, Ito T, Yoshida Y, Kobayashi Y, Katagiri H, Minamino T, Komuro I. Brain-derived neurotrophic factor protects against cardiac dysfunction after myocardial infarction via a central nervous system-mediated pathway. Arterioscler Thromb Vasc Biol. 2012; 32: 1902-1909.

22. Kermani P, Rafii D, Jin DK, Whitlock P, Schaffer W, Chiang A, Vincent L, Friedrich M, Shido K, Hackett NR, Crystal RG, Rafii S, Hempstead BL. Neurotrophins promote revascularization by local recruitment of TrkB+ endothelial cells and systemic mobilization of hematopoietic progenitors. J Clin Invest. 2005; 115: 653-663.

23. Hang $\mathrm{P}$, Zhao J, Cai B, Tian S, Huang W, Guo J, Sun C, Li Y, Du Z. Brain-derived neurotrophic factor regulates TRPC $3 / 6$ channels and protects against myocardial infarction in rodents. Int J Biol Sci. 2015; 11: 536-545.

24. Mellios N, Huang HS, Grigorenko A, Rogaev E, Akbarian S. A set of differentially expressed miRNAs, including miR-30a-5p, act as post-transcriptional inhibitors of BDNF in prefrontal cortex. Hum Mol Genet. 2008; 17: 3030-3042.

25. Marler KJ, Suetterlin P, Dopplapudi A, Rubikaite A, Adnan J, Maiorano NA, Lowe AS, Thompson ID, Pathania M, Bordey A, Fulga T, Van Vactor DL, Hindges R, Drescher U. BDNF promotes axon branching of retinal ganglion cells via miRNA-132 and p250GAP. J Neurosci. 2014; 34: 969-979.

26. Li M, Armelloni S, Zennaro C, Wei C, Corbelli A, Ikehata M, Berra S, Giardino L, Mattinzoli D, Watanabe S, Agostoni C, Edefonti A, Reiser J, Messa P, Rastaldi MP. BDNF repairs podocyte damage by microRNA-mediated increase of actin polymerization. J Pathol. 2015; 235: 731-744

27. Zhang X, Wang J, Zhou Q, Xu Y, Pu S, Wu J, Xue Y, Tian Y, Lu J, Jiang W, Du D. Brain-derived neurotrophic factor-activated astrocytes produce mechanical allodynia in neuropathic pain. Neuroscience. 2011; 199: 452-460.

28. Xu C, Hu Y, Hou L, Ju J, Li X, Du N, Guan X, Liu Z, Zhang T, Oin W, Shen N, Bilal MU, Lu Y, Zhang Y, Shan H. $\beta$-Blocker carvedilol protects cardiomyocytes against oxidative stress-induced apoptosis by up-regulating miR-133 expression. J Mol Cell Cardiol. 2014; 75: 111-121.

29. Dong DL, Chen C, Huo R, Wang N, Li Z, Tu YJ, Hu JT, Chu X, Huang W, Yang BF. Reciprocal repression between microRNA-133 and calcineurin regulates cardiac hypertrophy: a novel mechanism for progressive cardiac hypertrophy. Hypertension. 2010; 55: 946-952.

30. Hullinger TG, Montgomery RL, Seto AG, Dickinson BA, Semus HM, Lynch JM, Dalby CM, Robinson K, Stack C, Latimer PA, Hare JM, Olson EN, van 
Rooij E. Inhibition of miR-15 protects against cardiac ischemic injury. Circ Res. 2012; 110: 71-81.

31. Porrello ER, Mahmoud AI, Simpson E, Johnson BA, Grinsfelder D, Canseco D, Mammen PP, Rothermel BA, Olson EN, Sadek HA. Regulation of neonatal and adult mammalian heart regeneration by the miR-15 family. Proc Natl Acad Sci U S A. 2013; 110: 187-192.

32. Roy S, Khanna S, Hussain SR, Biswas S, Azad A, Rink C, Gnyawali S, Shilo S, Nuovo GJ, Sen CK. MicroRNA expression in response to murine myocardial infarction: miR-21 regulates fibroblast metalloprotease-2 via phosphatase and tensin homologue. Cardiovasc Res. 2009; 82: 21-29.

33. Chen $Y Q$, Wang $X X$, Yao XM, Zhang DL, Yang XF, Tian SF, Wang NS. MicroRNA-195 promotes apoptosis in mouse podocytes via enhanced caspase activity driven by BCL2 insufficiency. Am J Nephrol. 2011; 34: 549-559.

34. Varendi K, Kumar A, Härma MA, Andressoo JO. miR-1, miR-10b, miR-155, and miR-191 are novel regulators of BDNF. Cell Mol Life Sci. 2014; 71: 4443-4456.

35. Tian N, Cao Z, Zhang Y. MiR-206 decreases brain-derived neurotrophic factor levels in a transgenic mouse model of Alzheimer's disease. Neurosci Bull. 2014; 30: 191-197.

36. Varendi K, Mätlik K, Andressoo JO. From microRNA target validation to therapy: lessons learned from studies on BDNF. Cell Mol Life Sci. 2015; 72: 1779-1794.

37. Iekushi K, Seeger F, Assmus B, Zeiher AM, Dimmeler S. Regulation of cardiac microRNAs by bone marrow mononuclear cell therapy in myocardial infarction. Circulation. 2012; 125: 1765-1773, S1-S7. 